\title{
GENERALIZED INJECTIVITY AND CHAIN CONDITIONS
}

\author{
by NGUYEN V. DUNG
}

(Received 3 April, 1991)

Relationships between injectivity or generalized injectivity and chain conditions on a module category have been studied by several authors. A well-known theorem of Osofsky $[14,15]$ asserts that a ring all of whose cyclic right modules are injective is semisimple Artinian. Osofsky's proofs in $[14,15]$ essentially used homological properties of injective modules, and, later, her arguments were applied by other authors in their studies of rings for which cyclic right modules are quasi-injective, continuous or quasi-continuous (see e.g. $[1,10,12]$ ). Following [5] (cf. [4]), a module $M$ is called a CS-module if every submodule of $M$ is essential in a direct summand of $M$. In the recent paper [17], B. L. Osofsky and P. F. Smith have proved a very general theorem on cyclic completely CS-modules from which many known results in this area follow rather easily. In another direction, it was proved in [8] that a finitely generated quasi-injective module with ACC (respectively DCC) on essential submodules is Noetherian (respectively Artinian). This result was also extended to CS-modules in $[3,16]$, and weak CS-modules in [19].

While CS-modules are a generalization of injective modules, finitely generated CS-modules have an interesting property that their closed submodules are finitely generated, which can be regarded as a weak form of the Noetherian condition. This observation led to a study in [13] of modules all of whose closed submodules are finitely generated.

In this paper, we will be interested in a class of modules which contains both finitely generated CS-modules and modules with finite uniform dimension. A module $M$ will be called a CEF-module if every closed submodule of $M$ contains a finitely generated essential submodule, or, in other words, if every closed submodule of $M$ is essentially finitely generated. Similarly, a module $M$ is called a CEC-module if every closed submodule of $M$ contains a cyclic essential submodule, or, equivalently, every closed submodule of $M$ is essentially cyclic.

We will show that if $M$ is a finitely generated module such that for every nonzero submodule $N$ of $M, M / N$ and every cyclic submodule of $M / N$ is a direct sum of a CEC-module and a module with finite uniform dimension, then $M$ satisfies ACC on direct summands. A similar result holds also for CEF-modules. Thus we obtain a generalization of the main result in [9] on CS-modules. As a consequence, we get also a refinement of the Osofsky-Smith theorem in [17]. Further, it is proved that a CS-module $M$ for which $M / \operatorname{Soc}(M)$ satisfies $\mathrm{ACC}$ on direct summands is a direct sum of a semisimple module and a module with finite uniform dimension. Consequently, we obtain a partial extension of a result of Camillo and Yousif [3] from CS-modules to CEC-modules.

Among examples of CEC-modules, we could mention uniform modules, injective hulls of cyclic modules, or, more generally, any CS-module $M$ with a cyclic essential submodule $K$. Indeed, if $A$ is a closed submodule in $M$, then $A$ is a direct summand of $M$. Thus the projection of $K$ in $A$ is cyclic, and contains $K \cap A$ which clearly is essential in $A$. Hence $A$ contains a cyclic essential submodule. Similarly, examples of CEF-modules are

Glasgow Math. J. 34 (1992) 319-326. 
modules with finite uniform dimension, injective hulls of finitely generated modules, or any CS-module with a finitely generated essential submodule.

Throughout this paper, all rings considered are associative with identity and all modules are unitary right modules. For a module $M, \operatorname{Soc}(M)$ will denote the socle of $M$, and $M$ is semisimple if $M=\operatorname{Soc}(M)$. A submodule $N$ of $M$ is called essential in $M$ if $N \cap K \neq 0$ for every nonzero submodule $K$ of $M$. In this case, $M$ is called an essential extension of $N$. A submodule $C$ is called closed in $M$ if $C$ has no proper essential extensions in $M$. A module $M$ is said to have finite uniform dimension if $M$ does not contain an infinite direct sum of nonzero submodules. A module $N$ is called a subquotient of a module $M$ if $N$ is a submodule of a quotient of $M$.

We now state the main result of this paper.

THeOREM 1. Let $M$ be a finitely generated module such that for every nonzero submodule $A$ of $M, M / A$ and all cyclic submodules of $M / A$ are direct sums of $a$ CEC-module and a module with finite uniform dimension. Then $M$ satisfies $\mathrm{ACC}$ on direct summands.

To prove this theorem, we will adapt the techniques developed by Osofsky and Smith in [17]. First, we prove a lemma, which is of independent interest.

LEMma 2. Let $N$ be a CEF-module with the infinitely generated essential socle $S$ such that every finitely generated submodule of $S$ is a direct summand of $N$, and every cyclic submodule of $N$ is a direct sum of a CEF-module and a module with finitely generated socle. Then $N / S$ is not a CEC-module.

Proof. Suppose that $N / S$ is a CEC-module. Let $S=\bigoplus_{i=1}^{\infty} S_{i}$ such that all $S_{i}$ are infinitely generated. For each $i, S_{i}$ has a maximal essential extension $D_{i}$. Since $D_{i}$ is closed in $N, D_{i}$ contains a finitely generated essential submodule. Thus it is clear that $D_{i} \neq S_{i}$ for each $i$, so $D_{i}^{\prime}=\left(D_{i}+S\right) / S$ is nonzero for every $i$. Let $A^{\prime}$ be a maximal essential extension of $\bigoplus_{i=1}^{\infty} D_{i}^{\prime}$ in $N / S$. Since $N / S$ is a CEC-module, $A^{\prime}$ contains a cyclic essential submodule $E^{\prime}$. There exists a cyclic submodule $E$ of $N$ such that $(E+S) / S=E^{\prime}$. Since $E^{\prime}$ is essential in $A^{\prime}, C_{i}^{\prime}=E^{\prime} \cap D_{i}^{\prime}$ is nonzero for each $i$. Let $C_{i}$ be the inverse image of $C_{i}^{\prime}$ in $D_{i}$ under the canonical map. Then we have $S_{i} \subset C_{i} \subseteq D_{i}$, and clearly $C_{i}$ is not contained in $S$. Because $C_{i}^{\prime} \subseteq E^{\prime}$, we have

$$
C_{i} \subseteq E+S=E \oplus T
$$

for some submodule $T$ of $S$. If $C_{i} \cap E=0$ for some $i$, then $C_{i}$ is isomorphic to a submodule of $T$, thus $C_{i}$ is semisimple and so $C_{i}$ is contained in $S$, a contradiction. Therefore we have that for each $i C_{i} \cap E \neq 0$. But $C_{i}$ is an essential extension of $S_{i}$, so it follows that $S_{i} \cap E \neq 0$ for each $i$. Then we can take a nonzero simple submodule $V_{i}$ in $S_{i} \cap E$ for each $i$.

Since $E$ is cyclic, by assumption, $E=F \oplus K$, where $F$ is a CEF-module and $K$ has finitely generated socle. It is easy to see that $K$ is finitely generated semisimple. Let $V=\bigoplus_{i=1}^{\infty} V_{i}$ and $U=F \cap V$. Then $V=U \oplus X$, where $X$ is isomorphic to a submodule of $K$; hence $X$ is finitely generated. Thus $U$ is infinitely generated. Since $F$ is a CEF-module, and $U$ is semisimple, $U$ has a finitely generated essential extension $L$ in $F$. Clearly $L \neq U$, 
thus $L^{\prime}=(L+S) / S$ is nonzero, and $L^{\prime} \subset A^{\prime}$. Now we want to show that $L \cap\left(\bigoplus_{i=1}^{\infty} D_{i}\right) \subseteq S$. Since $S \subseteq \bigoplus_{i=1}^{\infty} D_{i}$, this would imply that $L^{\prime} \cap\left(\bigoplus_{i=1}^{\infty} D_{i}^{\prime}\right)=0$, a contradiction of the fact that $\bigoplus_{i=1}^{\infty} D_{i}^{\prime}$ is essential in $A^{\prime}$.

In fact, for each $n$, we have

$$
\begin{aligned}
& \left(L \cap \bigoplus_{i=1}^{n} D_{i}\right) \cap S=L \cap\left(\left(\bigoplus_{i=1}^{n} D_{i}\right) \cap S\right) \\
& =L \cap \bigoplus_{i=1}^{n} S_{i} \subseteq\left(\bigoplus_{i=1}^{\infty} V_{i}\right) \cap\left(\bigoplus_{i=1}^{n} S_{i}\right)=\bigoplus_{i=1}^{n} V_{i} .
\end{aligned}
$$

But $\bigoplus_{i=1}^{n} V_{i}$ is a direct summand of $N$ by assumption; together with the fact that $S$ is essential in $N$, it implies that $L \cap \bigoplus_{i=1}^{n} D_{i} \subseteq S$, for each $n$. Thus we have $L \cap \bigoplus_{i=1}^{\infty} D_{i} \subseteq S$ which gives us the desired contradiction. This completes the proof of Lemma 2.

LEMMA 3. Let $M$ be a CEF-module such that $M / \operatorname{Soc}(M)$ has finite uniform dimension. Then $M$ has finite uniform dimension.

Proof. It is enough to show that $S=\operatorname{Soc}(M)$ is finitely generated. Suppose that $S$ is infinitely generated; then we can write $S=\bigoplus_{i=1}^{\infty} S_{i}$, where each $S_{i}$ is infinitely generated. Since $M$ is a CEF-module, each $S_{i}$ has a maximal essential extension $D_{i}$ which contains a finitely generated essential submodule $B_{i}$. Then clearly $D_{i} \neq S_{i}$, and $\bigoplus_{i=1}^{\infty}\left(\left(D_{i}+S\right) / S\right)$ is an infinite direct sum in $M / S$, a contradiction.

We are now in a position to prove Theorem 1.

Proof of Theorem 1. Suppose that there exists an infinite ascending chain of direct summands $A_{i}$ of $M$ :

$$
A_{1} \subset A_{2} \subset \ldots \subset A_{i} \subset \ldots
$$

There is a direct summand $B_{1}$ of $M$ such that $M=A_{1} \oplus B_{1}$. Then it is clear that $A_{2}=A_{1} \oplus\left(A_{2} \cap B_{1}\right)$. Thus there is a submodule $B_{2}$ of $B_{1}$ such that $B_{1}=B_{2} \oplus\left(A_{2} \cap B_{1}\right)$. It follows that $M=A_{2} \oplus B_{2}$. Repeating this argument, we produce an infinite descending chain of direct summands $B_{i}$ of $M$ with

$$
B_{1} \supset B_{2} \supset \ldots \supset B_{i} \supset \ldots
$$

Let $B_{n}=C_{n+1} \oplus B_{n+1}$ with $n \geq 1$, and put $C_{1}=A_{1}$. Then we get an infinite sequence $\left\{C_{n}\right\}$ of direct summands $C_{n}$ of $M$, such that

$$
M=\left(\bigoplus_{i=1}^{n} C_{i}\right) \oplus B_{n}, \quad \text { and } \quad\left(\bigoplus_{j=n+1}^{\infty} C_{j}\right) \subseteq B_{n}
$$

for each $n \geq 1$. 
Since each $C_{i}$ is finitely generated, $C_{i}$ contains a maximal submodule $X_{i}$. Consider the quotient module $P=M / \bigoplus_{i=1}^{\infty} X_{i}$. Then we have $S=\left(\bigoplus_{i=1}^{\infty} C_{i}\right) /\left(\bigoplus_{i=1}^{\infty} X_{i}\right)$ is a semisimple submodule of $P$. Note that, by the construction, for each $n, S_{n}=\bigoplus_{i=1}^{n}\left(C_{i} / X_{i}\right)$ is a direct summand of $P$. It follows that every finitely generated submodule of $S$, being a direct summand of some $S_{n}$, must be a direct summand of $P$.

By hypothesis, $P=P_{1} \oplus D$, where $P_{1}$ is a CEC-module and $D$ has finite uniform dimension. Let $S^{\prime}=P_{1} \cap S$; then $S=S^{\prime} \oplus T$ for some submodule $T$ of $S$. Since $T$ is isomorphic to a submodule of $D, T$ is finitely generated. Thus $S^{\prime}$ is infinitely generated. Since $P_{1}$ is a CEC-module, it is easy to see that $S^{\prime}$ has a cyclic essential extension $L$ in $P_{1}$. Again by hypothesis, $L=N \oplus F$ such that $N$ is a CEC module and $F$ has finite uniform dimension. Let $Q=N \cap S^{\prime}$; then $Q$ is essential in $N$. Repeating the above argument, we can show that $Q$ is infinitely generated. It is also clear that every finitely generated submodule of $Q$ is a direct summand of $N$.

Now we have $N / Q=H \oplus G$, where $H$ is a CEC-module and $G$ has finite uniform dimension. We see that $N$ satisfies the conditions of Lemma 2, thus $N / Q$ cannot be a CEC-module, so $G$ must be nonzero. Since $G$ is cyclic, there is a cyclic submodule $N_{1}$ of $N$ such that $\left(N_{1}+Q\right) / Q \cong G$. Let $Q_{1}=N_{1} \cap Q$; then $Q_{1}=\operatorname{Soc}\left(N_{1}\right)$ and $N_{1} / Q_{1} \cong G$, so $N_{1} / Q_{1}$ has finite uniform dimension. By hypothesis, $N_{1}=N_{2} \oplus Y$ such that $N_{2}$ is a CEC-module and $Y$ has finite uniform dimension. Then

$$
N_{1} / Q_{1} \cong N_{2} / \operatorname{Soc}\left(N_{2}\right) \oplus Y / \operatorname{Soc}(Y) \text {. }
$$

and it follows that $N_{2} / \operatorname{Soc}\left(N_{2}\right)$ has finite uniform dimension. By Lemma 3, $\operatorname{Soc}\left(N_{2}\right)$ is finitely generated, hence $Q_{1}$ is finitely generated. Therefore $Q_{1}$ is a direct summand of $N$, and, since $Q_{1}$ is essential in $N_{1}$, it follows that $N_{1}=Q_{1}$, so $G=0$. This contradiction completes the proof of the theorem.

REMARK. We are unable to answer the following question. Let $M$ be a cyclic module such that every cyclic subquotient of $M$ is a direct sum of a CEC-module and a module with finitely generated socle. Does $M$ satisfy ACC on direct summands?

For CEF-modules, the following theorem can be obtained with a proof similar to that of Theorem 1 .

THEOREM 4. Let $M$ be a finitely generated module such that for every nonzero submodule $A$ of $M$, every finitely generated submodule of $M / A$ is a direct sum of a CEF-module and a module with finite uniform dimension. Then $M$ has ACC on direct summands.

Next we will derive some consequences of these results. The first corollary is the main result of [9], which is turn is a generalization of [6] and [7].

Corollary 5 (see [9]). Let $M$ be a cyclic module such that every cyclic subquotient of $M$ is a direct sum of a CS-module and a module with finite uniform dimension. Then $M$ is a finite direct sum of uniform modules.

Proof. By Theorem 1, $M$ has ACC on direct summands; thus $M$ is a finite direct sum of indecomposable submodules. Now the result follows from the fact that an indecomposable CS-module is uniform. 

[17].

The next result can be regarded as a refinement of the Osofsky-Smith theorem in

Corollary 6. Let $M$ be a cyclic module such that every cyclic subquotient of $M$ is a CEC-module. Then $M$ has ACC on direct summands.

COROLLARY 7. Let $M$ be a finitely generated module such that every finitely generated subquotient of $M$ is a CEF-module. Then $M$ has ACC on direct summands.

The following result is immediate from Corollaries 6 and 7.

COROLLARY 8. Let $R$ be a ring for which every cyclic (respectively finitely generated) right module is a CEC-module (respectively CEF-module). Then every cyclic (respectively finitely generated) right $R$-module satisfies $\mathrm{ACC}$ on direct summands.

If $R$ is a von Neumann regular ring, then every finitely generated right ideal of $R$ is principal. Thus, from the proof of Theorem 1 , we obtain

COROLlary 9. Let $R$ be a von Neumann regular ring. Then $R$ is semisimple Artinian if and only if every cyclic right $R$-module is a CEF-module.

In [14, Lemma 5], Osofsky proved that if $\left\{e_{i}\right\}_{i=1}^{\infty}$ is an infinite set of orthogonal idempotents in a von Neumann regular right self-injective ring $R$, then $R / \bigoplus_{i=1}^{\infty} e_{i} R$ is not an injective right $R$-module. From Lemma 2 we can obtain a related result.

COROLlaRY 10. Let $R$ be a von Neumann regular right self-injective ring. If $S=\operatorname{Soc}\left(R_{R}\right)$ is infinitely generated, then $(R / S)_{R}$ is not a CEF-module.

Proof. Let $E$ be the injective hull of $S$ in $R_{R}$; then the module $E_{R}$ satisfies the conditions of Lemma 2 . Note that every finitely generated submodule of $E_{R}$ is cyclic. From the proof of Lemma 2, it follows that $(E / S)_{R}$ is not a CEF-module. It is easy to prove that a direct summand of a CEF-module is again CEF, so we obtain that $(R / S)_{R}$ is not a CEF-module.

Modules with chain conditions on essential submodules have been studied extensively in recent years (see e.g. $[2,3, \mathbf{8}, \mathbf{1 1}, \mathbf{1 6}, \mathbf{1 8}, \mathbf{1 9}]$ ). Extending [8] and [11], Camillo and Yousif [3] showed that if $M$ is a CS-module such that $M / \operatorname{Soc}(M)$ has finite uniform dimension, then $M$ is a direct sum of a semisimple module and a module with finite uniform dimension. Note that a module with finite uniform dimension has ACC on direct summands. Now we shall extend the above result of Camillo and Yousif to CS-modules $M$ with $M / \operatorname{Soc}(M)$ satisfying $\mathrm{ACC}$ on direct summands. For our result, we need some lemmas, the first of which is elementary, so we omit the proof.

LemMA 11. For a module $M$ the following conditions are equivalent.

(a) $M$ satisfies ACC on direct summands.

(b) $M$ does not contain an infinite direct sum $\bigoplus_{i=1}^{\infty} A_{i}$ of submodules $A_{i}$, where $\bigoplus_{i=1}^{n} A_{i}$ is $a$ direct summand of $M$ for each $n \geq 1$.

LEMMA 12. Let $M$ be a module and $S=\operatorname{Soc}(M)$.

(a) If $A$ and $B$ are submodules of $M$ with $A \cap B=0$, then

$$
((A+S) / S) \cap((B+S) / S)=0 \text {. }
$$


(b) If $A$ is a direct summand of $M$, then $(A+S) / S$ is a direct summand of $M / S$.

(c) If $\bigoplus_{i \in I} A_{i}$ is a direct sum of submodules of $M$, then $\bigoplus_{i \in I}\left(\left(A_{i}+S\right) / S\right)$ is also a direct sum of submodules in $M / S$.

Proof. (a) Let $f: M \rightarrow M / S$ be the canonical map. Suppose that $A$ and $B$ are submodules of $M$ such that $A \cap B=0$. Set $\bar{V}=f(A) \cap f(B)$, then there exists a submodule $V \subseteq A$ such that $f(V)=\bar{V}$. Clearly $V \subseteq B+S=B \oplus T$ for some submodule $T \subseteq S$. Since $V \cap B=0$, it follows that $V$ is isomorphic to a submodule of $T$. Hence $V \subseteq S$ which implies that $\bar{V}=0$. Therefore we have $f(A) \cap f(B)=0$.

(b) Let $A$ be a direct summand of $M$. Then $M=A \oplus B$ for some submodule $B$ of $M$. Clearly $M / S=f(A)+f(B)$. By (a) we have $f(A) \cap f(B)=0$. Thus $f(A)$ is a direct summand of $M / S$.

(c) This is an immediate consequence of (a).

Following Smith [19], a module $M$ will be called almost semisimple if $M$ has essential socle and every finitely generated semisimple submodule of $M$ is closed in $M$.

PROPOSITION 13. Let $M$ be a CS-module such that $M / \operatorname{Soc}(M)$ has ACC on direct summands. Then $M$ is a direct sum of a semisimple module and a module with finite uniform dimension.

Proof. Let $S=\operatorname{Soc}(M)$. Then $M=M_{1} \oplus M_{2}$, where $S$ is essential in $M_{1}$. Clearly $M_{2}$ is isomorphic to a direct summand of $M / S$, thus by hypothesis $M_{2}$ has ACC on direct summands. Hence $M_{2}$ is a finite direct sum of indecomposable submodules, and, since $M_{2}$ is CS, $M_{2}$ is a finite direct sum of uniform modules. Therefore, without loss of generality, we may assume that $M$ has essential socle.

Now we show that $M$ is a direct sum of an almost semisimple module and a module with finite uniform dimension. If $M$ is not almost semisimple, there exists a finitely generated submodule $S_{1}$ of $S$ such that $S_{1}$ is not closed in $M$. Then $S_{1}$ is essential in a direct summand $A_{1}$ of $M$, and $A_{1} \neq S_{1}$. Let $M=A_{1} \oplus B_{1}$. If $B_{1}$ is not almost semisimple, $B_{1}$ contains a finitely generated semisimple submodule $S_{2}$ which is not closed in $B_{1}$. Thus $S_{2}$ is essential in a direct summand $A_{2}$ of $B_{1}$, and $A_{2} \neq S_{2}$. Note that $A_{1} \oplus A_{2}$ is a direct summand of $M$. Repeat this process; it produces a direct sum $A_{1} \oplus A_{2} \oplus A_{3} \oplus \ldots$ of submodules $A_{i}$ of $M$ such that $A_{i}$ has finite uniform dimension and $A_{i}$ is not semisimple for each $i \geq 1$, and furthermore $\bigoplus_{i=1}^{n} A_{i}$ is a direct summand of $M$ for all $n \geq 1$. By Lemma 12 ,

$$
\left(\left(A_{1}+S\right) / S\right) \oplus\left(\left(A_{2}+S\right) / S\right) \oplus \ldots
$$

is a direct sum in $M / S$, and $\bigoplus_{i=1}^{n}\left(\left(A_{i}+S\right) / S\right)$ is a direct summand of $M / S$ for all $n \geq 1$. By Lemma 11 , this process must stop. Therefore $M$ is a direct sum of an almost semisimple module $N$ and a module $F$ with finitely generated essential socle. Clearly $N$ is a CS-module and $N / \operatorname{Soc}(N)$ has ACC on direct summands.

Next we show that $N$ is semisimple. Let $E$ be a finitely generated submodule of $N$. Then $E$ is essential in a direct summand $H$ of $N$. Suppose that $T=\operatorname{Soc}(E)$ is infinitely generated. We claim that $T$ contains an infinitely generated submodule which is closed in 
$H$. Assume that it is not so. Take an infinitely generated submodule $T_{1}$ of $T$ such that $T / T_{1}$ is infinitely generated. Then $H=C_{1} \oplus D_{1}$, where $T_{1}$ is essential in $C_{1}$. Clearly $\operatorname{Soc}\left(D_{1}\right)$ is infinitely generated. Take an infinitely generated submodule $T_{2}$ of $\operatorname{Soc}\left(D_{1}\right)$ such that $\left(\operatorname{Soc}\left(D_{1}\right)\right) / T_{2}$ is infinitely generated. Then $T_{2}$ is essential in a direct summand $C_{2}$ of $H$. Continuing in this manner, we get an infinite direct sum $C_{1} \oplus C_{2} \oplus C_{3} \oplus \ldots$ of $H$ such that $C_{i}$ is not semisimple and $\bigoplus_{i=1}^{n} C_{i}$ is a direct summand of $H$ for all $n \geq 1$. Since $H / \operatorname{Soc}(H)$ has also $\mathrm{ACC}$ on direct summands, Lemma 12 gives us a contradiction. Thus $T$ contains an infinitely generated submodule $K$ which is closed in $H$. Then $K$ is a direct summand of $H$, and hence of $E$, so $K$ is finitely generated. This contradiction shows that $T=\operatorname{Soc}(E)$ must be finitely generated. Because $N$ is $C S$ and almost semisimple, $T$ is a direct summand of $N$. But $T$ is essential in $E$, so we get that $T=E$, thus $E$ is semisimple. Therefore $N$ is semisimple which completes the proof.

REMARK. In [19] Smith introduced weak CS-modules as modules in which every semisimple submodule is essential in a direct summand. By [19, Corollary 2.7], if $M$ is a weak CS-module such that $M / \operatorname{Soc}(M)$ has finite uniform dimension, then $M$ is a direct sum of a semisimple module and a module with finite uniform dimension. Also, Osofsky [16] studied CS-modules satisfying $\aleph$-chain conditions on essential submodules, for an infinite cardinal $\aleph$. Thus, it might be interesting to investigate CS or weak CS-modules $M$ such that $M / \operatorname{Soc}(M)$ satisfies $N$-chain conditions on direct summands, for an infinite cardinal $\mathrm{K}$.

Extending the Osofsky-Smith theorem [17], Camillo and Yousif [3] proved that if $M$ is a cyclic CS-module such that all cyclic singular subquotients of $M$ are CS-modules, then $M$ has finite uniform dimension. As an application of Proposition 13, we can now get a partial generalization of this last result.

Proposition 14. Let $M$ be a CS-module with the essential socle $S$ such that $M / S$ is cyclic and all cyclic singular subquotients of $M$ are CEC-modules. Then $M$ is a direct sum of a semisimple module and a module with finite uniform dimension.

Proof. Clearly every cyclic subquotient of $M / S$ is a CEC-module. Thus, by Corollary $6, M / S$ has $\mathrm{ACC}$ on direct summands. The result follows now by Proposition 13.

ACKNOwLEDGements. This paper was written during a stay of the author at the University of Murcia, supported by the Spanish Ministry of Education and Science. The author wishes to express his warmest thanks to the Mathematics Department for its hospitality, and to Professor J. L. Gómez Pardo for his kind help.

\section{REFERENCES}

1. J. Ahsan, Rings all of whose cyclic modules are quasi-injective. Proc. London Math Soc. (3) 27 (1973), 425-439.

2. E. P. Armendariz, Rings with dcc on essential left ideals. Comm. Algebra 8 (1980), 299-308.

3. V. Camillo and M. F. Yousif, CS-modules with acc or dcc on essential submodules, Comm. Algebra 19 (1991), 655-662.

4. A. W. Chatters and C. R. Hajarnavis, Rings in which every complement right ideal is a direct summand. Quart. J. Math. Oxford (2) 28 (1977), 61-80. 
5. A. W. Chatters and S. M. Khuri, Endomorphism rings of modules over non-singular CS-rings. J. London Math. Soc. (2) 21 (1980), 434-444.

6. Dinh Van Huynh and Nguyen V. Dung, A characterization of Artinian rings. Glasgow Math. J. 30 (1988), 67-73.

7. Dinh Van Huynh, Nguyen V. Dung and P. F. Smith, A characterization of rings with Krull dimension. J. Algebra 132 (1990), 104-112.

8. Dinh Van Huynh, Nguyen V. Dung and R. Wisbauer, Quasi-injective modules with acc or dcc on essential submodules. Arch. Math. 53 (1989), 252-255.

9. Dinh Van Huynh, Nguyen V. Dung and R. Wisbauer, On modules with finite uniform and Krull dimension. Arch. Math. 57 (1991), 122-132.

10. V. K. Goel and S. K. Jain, $\pi$-injective modules and rings whose cyclics are $\pi$-injective. Comm. Algebra 6 (1978), 59-73.

11. S. K. Jain, S. R. López-Permouth and S. T. Rizvi, Continuous rings with acc on essentials are Artinian. Proc. Amer. Math. Soc. 108 (1990), 583-586.

12. S. K. Jain and S. Mohamed, Rings whose cyclic modules are continuous. Indian Math. Soc. Journal 42 (1978), 197-202.

13. Nguyen V. Dung, Modules whose closed submodules are finitely generated. Proc. Edinburgh Math. Soc. 34 (1991), 161-166.

14. B. L. Osofsky, Rings all of whose finitely generated modules are injective. Pacific J. Math. 14 (1964), 645-650.

15. B. L. Osofsky, Noninjective cyclic modules. Proc. Amer. Math. Soc. 19 (1968), 1383-1384.

16. B. L. Osofsky, Chain conditions on essential submodules. Proc. Amer. Math. Soc. 114 (1992), 11-19.

17. B. L. Osofsky and P. F. Smith, Cyclic modules whose quotients have all complement submodules direct summands. J. Algebra, 139 (1991), 342-354.

18. S. S. Page and M. F. Yousif, Relative injectivity and chain conditions. Comm. Algebra 17 (1989), 899-924.

19. P. F. Smith, CS-modules and weak CS-modules, in Non-commutative ring theory, Lecture Notes in Mathematics No. 1448 (Springer-Verlag, 1990), 99-115.

Departamento de Matemáticas

Universidad de MURCIA

30071 MurCIA, Spain
Permanent address:

INSTITUTE OF MATHEMATICS P.O. BoX 631 Bo Ho

Hanoi, Vietnam. 